\title{
Review of High-resolution Retinal Prosthetic System for Vision Rehabilitation: Our Perspective Based on 18 Years of Research
}

\author{
Sangmin Lee, ${ }^{1 \dagger}$ Kyo-in Koo, ${ }^{2 \dagger}$ Hyoungho Ko, ${ }^{3 \dagger}$ \\ Jong-Mo Seo, ${ }^{4,5}$ and Dong-il "Dan" Cho ${ }^{5 *}$ \\ ${ }^{1}$ Department of Biomedical Engineering, Kyung Hee University, \\ 26 Kyungheedae-ro, Dongdaemun-gu, Seoul 02477, Republic of Korea \\ ${ }^{2}$ Department of Biomedical Engineering, University of Ulsan, \\ 93 Daehak-ro, Nam-gu, Ulsan 44610, Republic of Korea \\ ${ }^{3}$ Department of Electronics Engineering, Chungnam National University, \\ 99 Daehak-ro, Yuseong-gu, Daejeon 34134, Republic of Korea \\ ${ }^{4}$ Department of Ophthalmology, School of Medicine, Seoul National University, \\ 103 Daehak-ro Jongno-gu, Seoul 03080, Republic of Korea \\ ${ }^{5}$ Department of Electrical and Computer Engineering, Seoul National University, \\ 1 Gwanak-ro, Gwanak-gu, Seoul 08826, Republic of Korea
}

(Received January 9, 2018; accepted January 25, 2018)

Keywords: retinal prosthesis, vision rehabilitation, electrical stimulation, high-resolution microelectrode array, current stimulator

Retinal degenerative diseases result in a progressive degeneration of photoreceptors in the retina and eventually lead to complete blindness. Among the many approaches tested, electrical stimulation of the remaining retinal neurons has shown the most promising results for vision rehabilitation of the blind. However, to improve the performance of retinal prosthetic systems, the fabrication of a device with a large number of stimulation electrodes is essential. For the successful development of a high-resolution prosthetic device, it is necessary to conduct highly collaborative research on microelectrode arrays and stimulation circuits, as well as verification of this research in animal experiments. In this paper, the requirements for a high-resolution retinal prosthetic system are explained, and then research efforts from around the world including the strategies of our research group to make major breakthroughs in artificial retina research are reviewed in detail.

\section{Introduction}

Retinal degeneration, such as retinitis pigmentosa (RP) and age-related macular degeneration (ARMD), is a condition that affects the photoreceptors in the retina and causes progressive loss of vision. ${ }^{(1-3)}$ These diseases cause the progressive degeneration of photoreceptors; however significant numbers of inner retinal cells, including bipolar and ganglion cells, continue to function even in the final stages of such diseases. ${ }^{(4,5)}$ Treatments such as gene therapy, ${ }^{(6)}$ drug medication, ${ }^{(7)}$ and cell transplantation ${ }^{(8)}$ have been reported to recover the degenerated

\footnotetext{
*Corresponding author: e-mail: dicho@snu.ac.kr

${ }^{\dagger}$ Co-first authors

http://dx.doi.org/10.18494/SAM.2018.1895
} 
photoreceptors, but those treatments have not shown satisfactory results. Therefore, electrical stimulation of the remaining retinal cells to evoke action potentials has been researched as an alternative to restore a limited form of vision in patients with retinal degenerative diseases. Clinical studies have shown that induced action potentials from the electrical stimulation of the surviving cells are propagated through the nerve to the visual cortex of the brain. ${ }^{(9,10)}$ In a normal retina, the photoreceptors modulate the released neurotransmitters in response to light and stimulate the bipolar and ganglion cell layers. ${ }^{(11)}$ For electrical stimulation methods, a microelectrode array (MEA) is used to replace the functions of degenerated photoreceptors by delivering electrical signals to the surviving inner cell layers. ${ }^{(12,13)}$ Images from an external camera are converted into electrical signals by processing units, and transmitted to the retinal network through the embedded MEA in the patient's eye, which electrically stimulate the surviving inner neurons. Figure 1 shows a schematic diagram of the conventional retinal prosthetic system consisting of an external camera, signal processing circuitry, and MEA.

In electrical stimulation methods, a large number of microelectrodes are required. ${ }^{(14)}$ However, the number of microelectrodes is limited by the overall size of the MEAs, which is determined by the area of the retina. Generally, an electrical stimulation method using current injection requires high output voltage compliance. Current amplitude ranging from a few tens of microamperes to a few hundreds of microamperes is required to evoke and increase the number of neuronal spikes of the retinal cells. ${ }^{(15)}$ The decreased surface area of the single microelectrode required for the high-resolution MEA leads to a higher electrode impedance, which limits the amplitude of the injection current. ${ }^{(16)}$ Therefore, to inject a sufficiently large current into high-resolution MEAs, research on microelectrode impedance and current stimulation circuits is necessary.

In this paper, we review the implementations of high-resolution retinal prosthetic systems. First, the high-resolution system requirements and the achievements of research groups from around the world are briefly reviewed. Then, the methodologies and results of our research group in high-resolution artificial retina research from the past 18 years are explained and summarized in detail. The development of three-dimensional (3D) microelectrodes to achieve

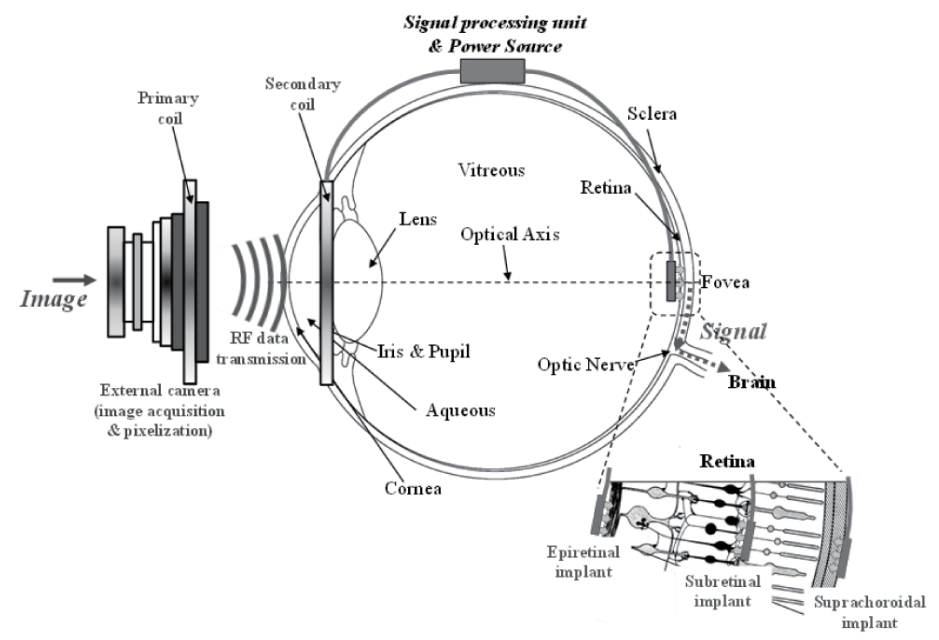

Fig. 1. Schematic of the conventional retinal prosthetic system. 
low interface impedance and high spatial resolution without reducing the effective surface area is summarized in Sect. 2. The development of current stimulation circuits to drive a large number of microelectrodes and the experimental results from animals are reviewed in detail in Sects. 3 and 4, respectively.

\section{High-resolution Microelectrode Arrays}

\subsection{Microelectrode array}

Although it is difficult to compare the resolution of a digital camera and that of a human eye, it has been widely suggested that the highest density region of the human eye corresponds to one hundred million pixels. In fact, the retina of the human eye has about 120 million rod cells, 6 million cone cells, and 1.3 million ganglion cells. Considering the size of the human eye, the single pixel size of retinal prostheses would be less than $20 \mu \mathrm{m}$. Therefore, it is not easy to restore complete visual acuity of a normal eye using the technologies available today.

Recognizing objects mainly relies on a spatial resolution. A visual angle of $0.5^{\circ}$ corresponds to a visual acuity of $1 / 35$, at which clinical tests can begin. ${ }^{(17)}$ Therefore, for retinal implants to restore vision, a minimum visual angle of $0.5^{\circ}$ is the goal. For daily-life activities, including eating, washing, and navigation, higher-order visual angles must be achieved. The minimum spatial resolution required for artificial vision to be useful can be derived using psychophysical methods. ${ }^{(18-20)}$ Sommerhalder et al. reported that at least 300 sampling points are necessary for near-perfect reading. ${ }^{(18)}$ Cha et al. reported that an additional 200 sampling points are needed for safe navigation in outdoor environments. ${ }^{(19)}$ The number of sampling points refers to the number of stimulation electrodes, which means that at least 500 microelectrodes should be placed in a single MEA for practical use to restore limited vision.

Cao et al. simulated the effects of various 3D microelectrodes, and compared them with two-dimensional (2D) microelectrodes. ${ }^{(21)}$ Heuschkel et al. reported a mountain-like 3D MEA on a rigid substrate, ${ }^{(22)}$ and Wang et al. fabricated a mountain-like 3D MEA on a flexible parylene substrate. ${ }^{(23)}$ Rajaraman et al. developed a cone-structured 3D MEA for in vitro brain slice recording. ${ }^{(24)}$ Bhandari et al. reported a convoluted-shaped 3D MEA on a rigid substrate. ${ }^{(25)}$ Hungar et al. developed a simple post-shaped 3D MEA on polyimide, ${ }^{(26)}$ and Kim et al. reported a mushroom-shaped 3D MEA on polyimide for retinal prostheses. ${ }^{(27)}$ Scribner et al. developed a convoluted 3D MEA on a rigid substrate, ${ }^{(28)}$ and Kusko et al. fabricated several noteworthy 3D MEAs on a rigid substrate. ${ }^{(29)}$ Djilas et al. reported a well-shaped MEA on a flexible substrate, ${ }^{(30)}$ and Xiang et al. fabricated a sharp MEA on a flexible substrate and recorded in vivo the neuronal signal of a rat brain. ${ }^{(31)}$

Our group has reported an arrowhead-shaped 3D MEA on a flexible polyimide substrate for retinal prostheses. ${ }^{(16,32-37)} 3 \mathrm{D}$ electrodes are expected to approach the target cells in the retina more closely than $2 \mathrm{D}$ electrodes. Also, the flexible polyimide substrate is adapted for conformal attachment to the spherical structure of the eye. In this research, using a silicon (Si) mold, an arrowhead-shaped electrode is fabricated. The arrowhead structure is capable of penetrating the inner limiting membrane in the retina with less invasiveness than the blunt end of a simple post structure. Figure 2(a) shows the fabrication processes of the arrowhead- 


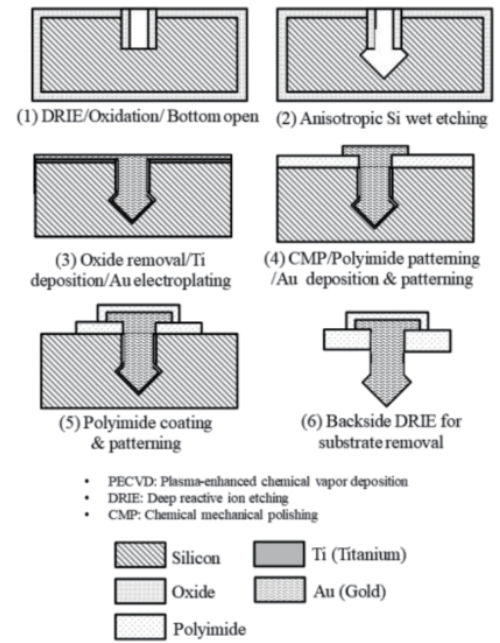

(a)

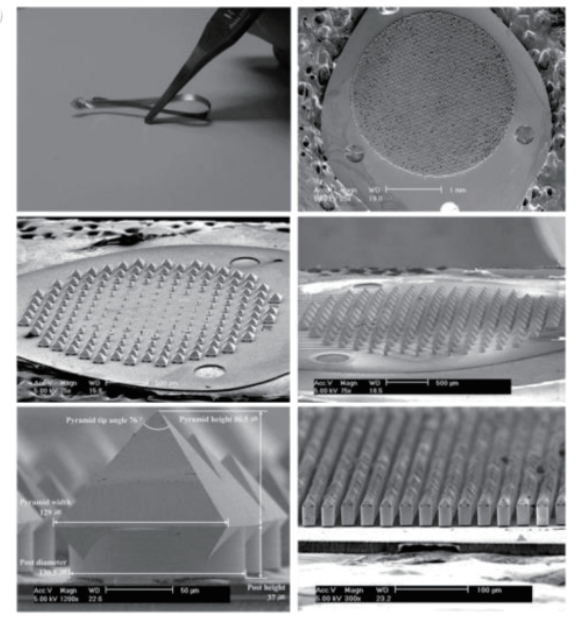

(b)

Fig. 2. Arrowhead-shaped MEA. ${ }^{(32)}$ (a) Fabrication process. (b) Fabrication results.

shaped microelectrode on the polyimide substrate. The base of each individual electrode is defined by silicon-dioxide patterning on a (100)-single-crystalline Si wafer. The height of the post structure part of each individual electrode is determined by a subsequent deep reactiveion etching (DRIE) process. For passivation of the side wall during the subsequent wet etching, a thermal oxidation process is performed. After the bottom Si layer is exposed by the bottom oxidation layer etching, alkaline wet etching reveals (111)-planes resulting in an octahedral structure. After the Si mold fabrication, all the films are removed, and then the gold (Au) seed layer is sputtered. Using electroplating, the Si mold is filled with a noble metal. The excess metal is removed by a chemical mechanical polishing (CMP) process. On the polished surface, the polyimide body layer and the conductive metal layer are patterned. Finally, the whole $\mathrm{Si}$ substrate is etched away from the opposite side. The fabricated MEAs, which have uniform, convex, and concave arrowhead-shaped electrodes, are shown in Fig. 2(b).

\subsection{Electrical and mechanical evaluation of MEAs}

To evaluate the advantage of 3D microelectrodes over 2D microelectrodes, two types of MEAs were fabricated. ${ }^{(16,36)}$ The first type of MEA consists of $2 \mathrm{D}$ circular microelectrodes with electrode diameters of 25, 50, and $75 \mu \mathrm{m}$. The second type of MEA consists of 3D arrowhead-shaped microelectrodes with base diameters of 25, 50, and $75 \mu \mathrm{m}$. The fabricated MEAs are wire-bonded to a printed circuit board (PCB) to measure the electrode-electrolyte interface impedance and current injection properties.

The electrode-electrolyte interface impedance measurement results are shown in Fig. 3(a). As the effective surface area increases, the impedance at $1 \mathrm{kHz}$ is decreased. As a consequence, the 3D arrowhead-shaped microelectrode with a base diameter of $25 \mu \mathrm{m}$ has a similar interface impedance to a $2 \mathrm{D}$ circular microelectrode with a base diameter of $75 \mu \mathrm{m}$. To evaluate the maximum current injection limit, each MEA is connected to the current stimulator, and the 

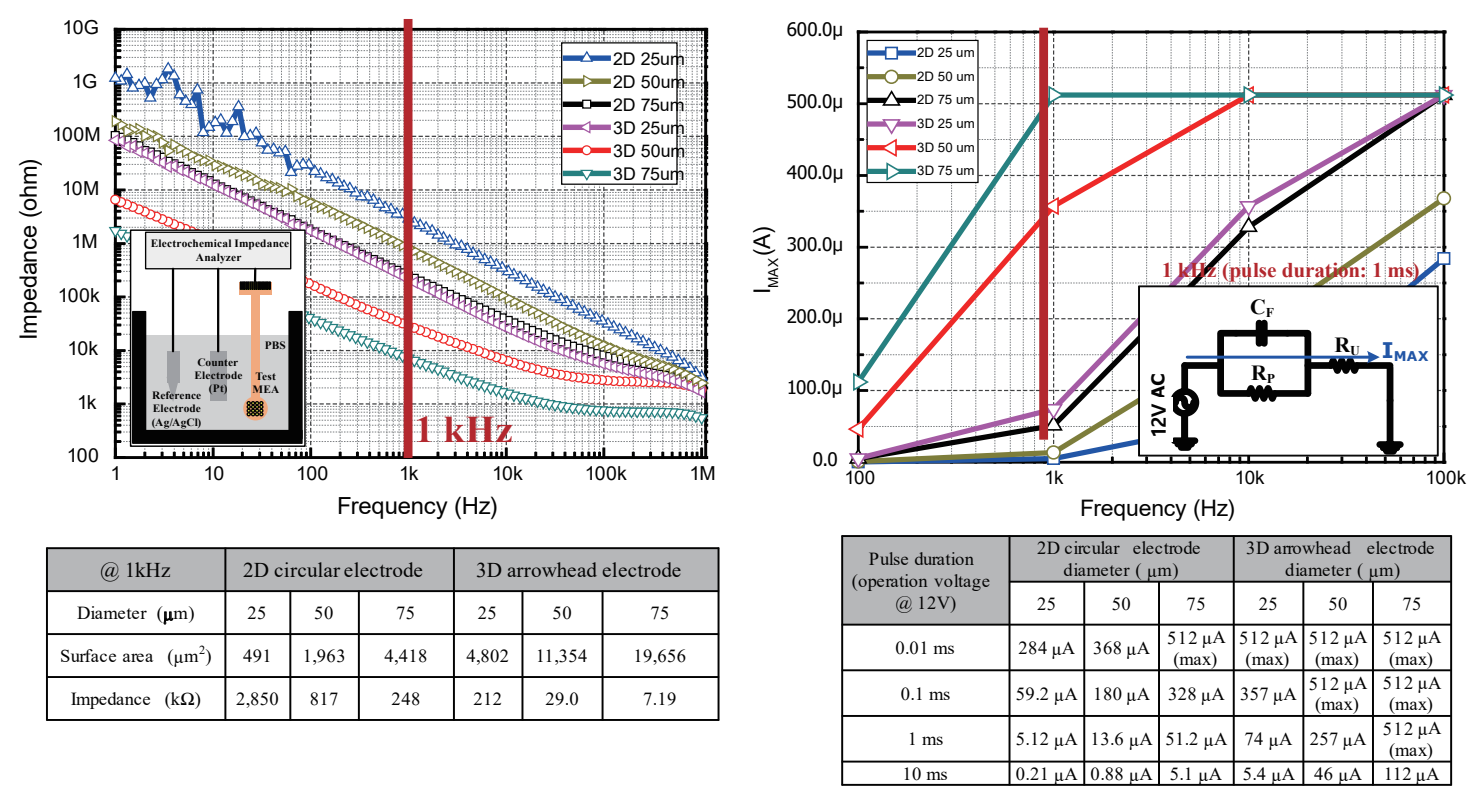

(a)

(b)

Fig. 3. (Color online) Electrical properties of 2D and 3D MEAs. ${ }^{(36)}$ (a) Electrode-electrolyte interface impedance. (b) Maximum current.

stimulation current is measured at $100 \mathrm{~Hz}, 1 \mathrm{kHz}, 10 \mathrm{kHz}$, and $100 \mathrm{kHz}$. The maximum current that the stimulator can generate is $512 \mu \mathrm{A}$, which is large enough to stimulate the ganglion cells in the retina. ${ }^{(15)}$ The results are shown in Fig. 3(b). The maximum current injection limit of the $3 \mathrm{D}$ microelectrode is much higher than that of the $2 \mathrm{D}$ microelectrode because of the larger effective surface area. Also, the results of the 2D microelectrode with a base diameter of $75 \mu \mathrm{m}$ and the 3D microelectrode with a base diameter of $25 \mu \mathrm{m}$ show similar results, which indicate that highly densified MEAs can be implemented using 3D microelectrodes.

Mechanical evaluation was performed to characterize the maximum vertical and horizontal stresses. ${ }^{(37)}$ The threshold force for the mechanical durability in the vertical direction can be seen in Fig. 4(a). The tip of the microelectrode is partially broken under a force of $1.470 \mathrm{~N}$. It is observed that all microelectrodes are entirely preserved without any mechanical damage when the applied force does not exceed $0.8165 \mathrm{~N}$. In the horizontal direction, the microelectrode is entirely broken when the applied mechanical force in the horizontal direction increases to up to 0.2068 N, as shown in Fig. 4(b).

\subsection{MEA combined with silicon nanowires}

As previously mentioned, a high-resolution system is required to provide a high-quality image in retinal prostheses. In the case of high-resolution retinal prosthesis systems that use external cameras, the electrical interface becomes complicated mainly because of the wiring complexity. ${ }^{(38)}$ Therefore, many commercialized retinal prostheses that use an external camera, such as the Argus II system (Second Sight Medical Products Inc., United States) ${ }^{(39)}$ or EpiRet3 


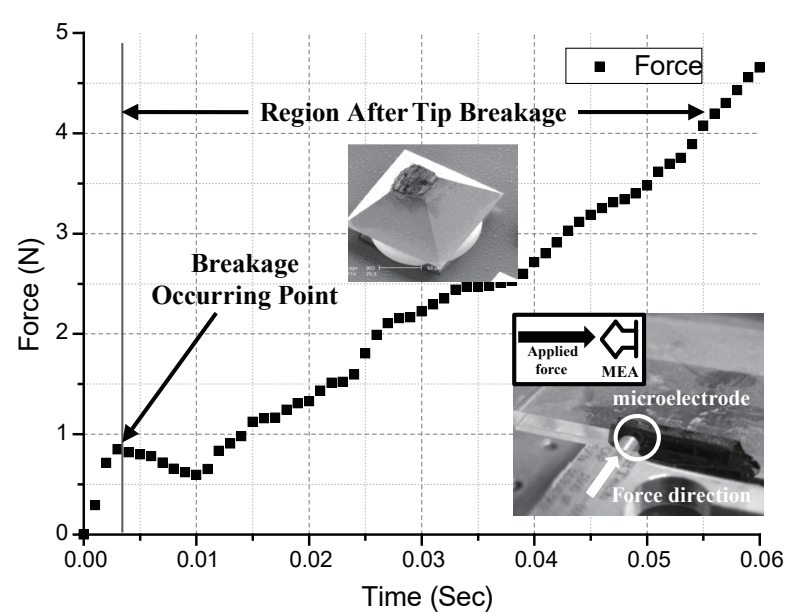

(a)

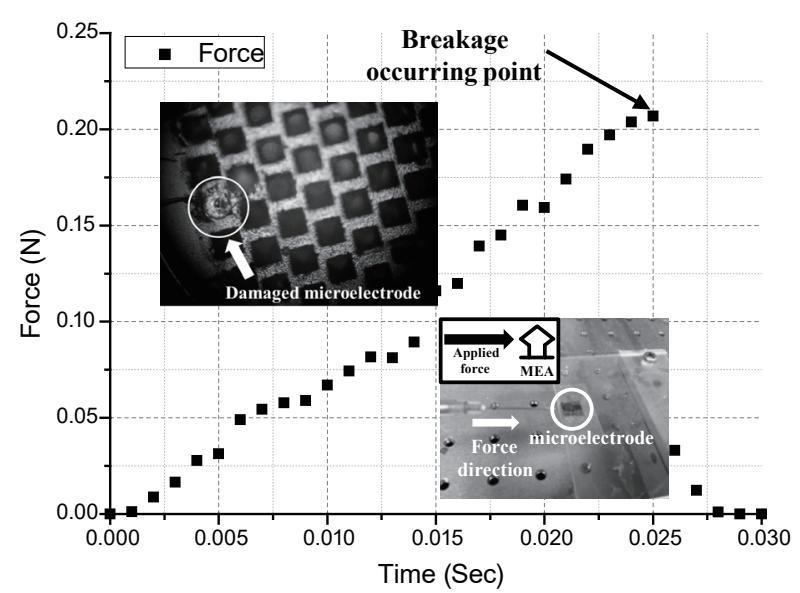

(b)

Fig. 4. Mechanical properties of 3D MEA. ${ }^{(37)}$ (a) Vertical direction. (b) Horizontal direction.

(EpiRet GmbH, Germany), ${ }^{(40)}$ are implemented with a small number of channels. On the other hand, retinal prosthetic devices with embedded photodetectors for image perception, such as the 'artificial silicon retina' (US Optobionics, United States) ${ }^{(41)}$ or Alpha IMS (German Retina Implant AG, Germany) ${ }^{(12)}$ have a relatively large number of channels owing to the collocation of the photodetector, stimulation circuit, and electrodes in a single channel. It has been reported that photodetector-embedded devices can easily solve the wiring problem of high-resolution retinal prosthetic systems. However, there remain problems to be solved, such as the device flexibility, overheating of the implanted stimulator chip, and low biocompatibility of the device material. $^{(42-44)}$

In our previous reports, a novel retinal stimulation device integrated with silicon nanowire (SiNW)-based field-effect transistor (FET) switches and photodetectors was demonstrated. ${ }^{(38,45-49)}$ The SiNWs are fabricated by a top-down method for wafer-level transfer on a flexible substrate, which can achieve very thin MEAs without compromising device flexibility. As shown in Fig. 5(a), the first type uses a SiNW FET as an active pixel switch, which is used for array addressing. From the two-dimensional array addressing method, only $M+N$ external wires are implemented for driving $M \times N$ microelectrodes. The second type uses the SiNW-based photodetection circuit consisting of a voltage divider and a current driver, as shown in Fig. 5(b). In a voltage divider, the SiNW FET and the SiNW PD are connected in series, which results in output voltage variation due to changes in the external light intensity. In the current driver, the SiNW FET and microelectrode are connected in series. The stimulation current is delivered to the microelectrode via SiNW FET, in which the current level is controlled by the output voltage of the voltage divider.

\section{Current Stimulators}

With the help of advances in high-voltage technology, many kinds of neural stimulators have been reported. One of the most important functions of neural stimulators is to induce 


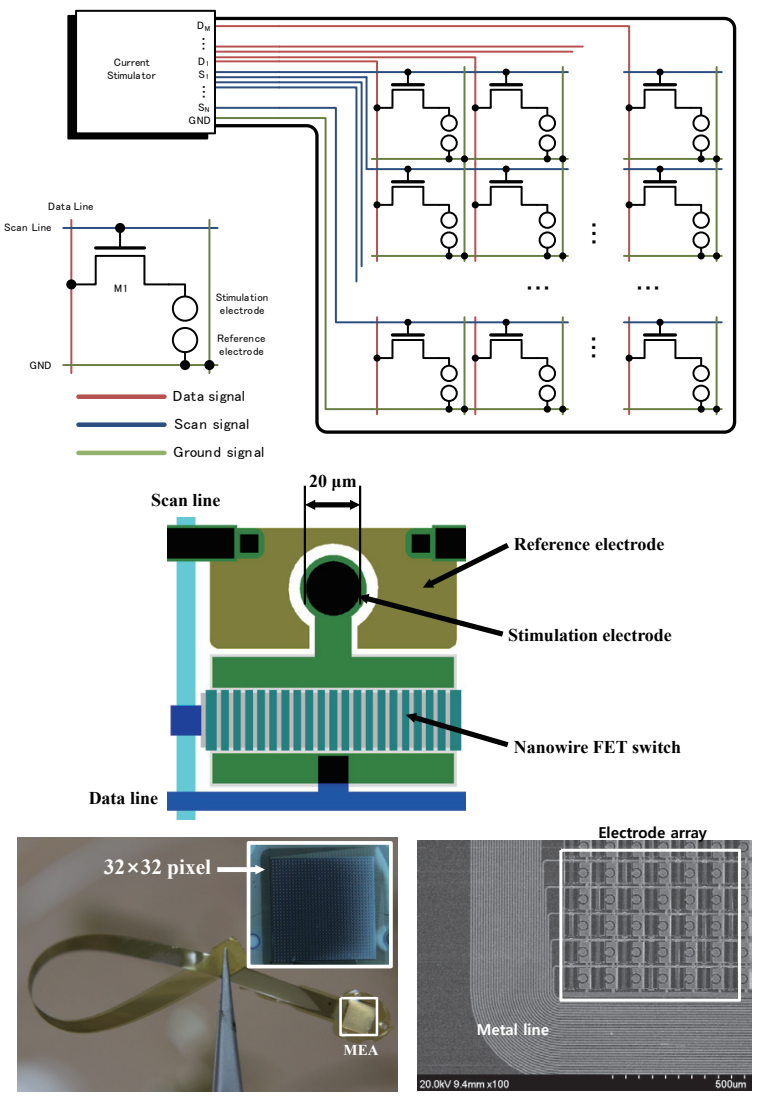

(a)
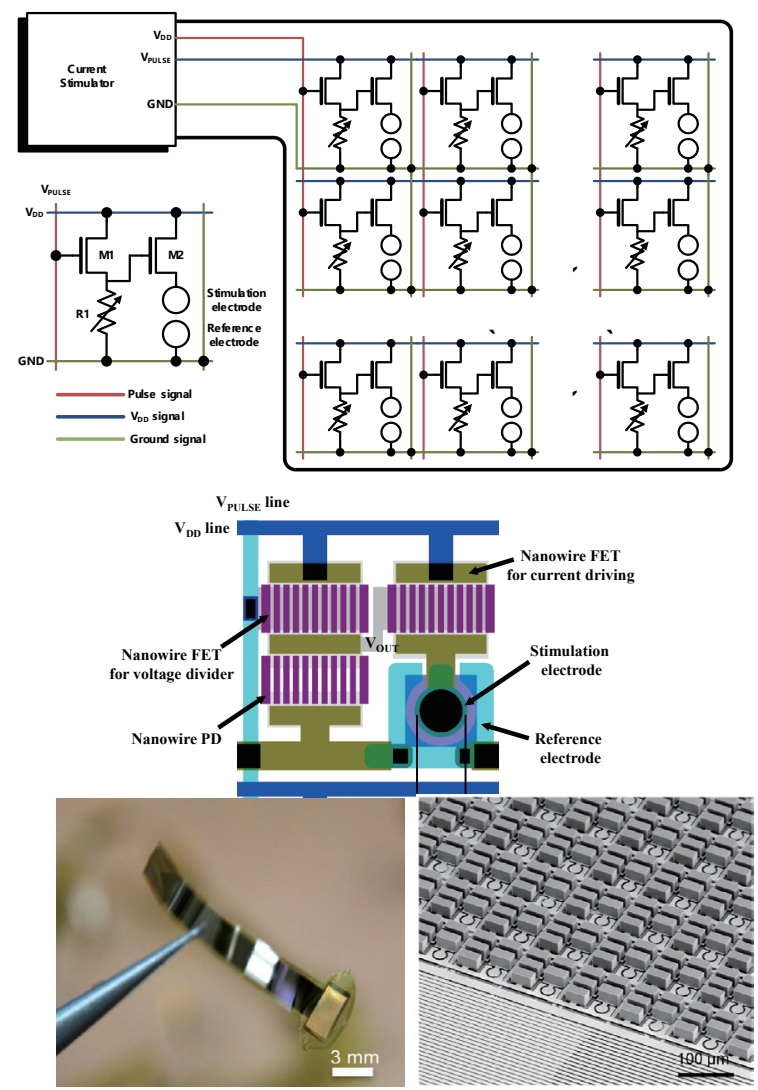

(b)

Fig. 5. (Color online) SiNW-based MEA. (a) First type (MEA with 2D array addressing). ${ }^{(38)}$ (b) Second type (MEA with photodetector). ${ }^{(46)}$

neural responses by delivering/injecting charge into nerve cells via electrodes. In most neural stimulators, a high-voltage supply is used because high-voltage compliance is required owing to the high electrode impedance. In the high-voltage stimulators, ensuring long-term stimulation safety is very important. As a result of current stimulation, tissue damage can occur in three possible ways: overstimulation, temperature rise of the tissue, and DC current flow into tissue. ${ }^{(50)}$

In terms of neural stimulators, stimulation safety is related to two main factors: power dissipation at tissue and by implanted electronics, and charge balancing (zero net remaining charge after biphasic current stimulation). A rise in temperature of $1-2{ }^{\circ} \mathrm{C}$ above the normal body temperature could lead to tissue damage; ${ }^{(51)}$ therefore, the total power dissipation of the implanted stimulator should be less than tens of $\mathrm{mW}$ to prevent an excessive rise in temperature. Maintaining charge balance during electrical stimulation is also important. The matched biphasic current pulses have often been used in electrical stimulation, as shown in Fig. 6(a) ${ }^{(52)}$ because the total amount of the delivered charge can be controlled by adjusting the pulse duration of the anodic and cathodic pulses. For a stimulator in a cochlear implant, the residual charge (residual DC current) should be kept below $100 \mathrm{nA} .{ }^{(53)}$ The typical circuit implementation of the biphasic current driver is shown in Fig. 2(b). ${ }^{(54)}$ The biphasic current 


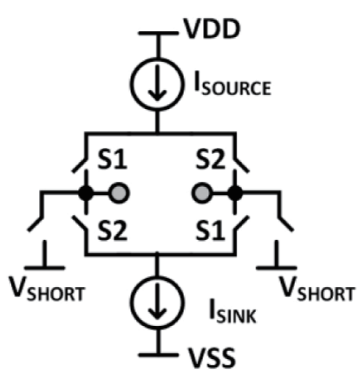

(a)

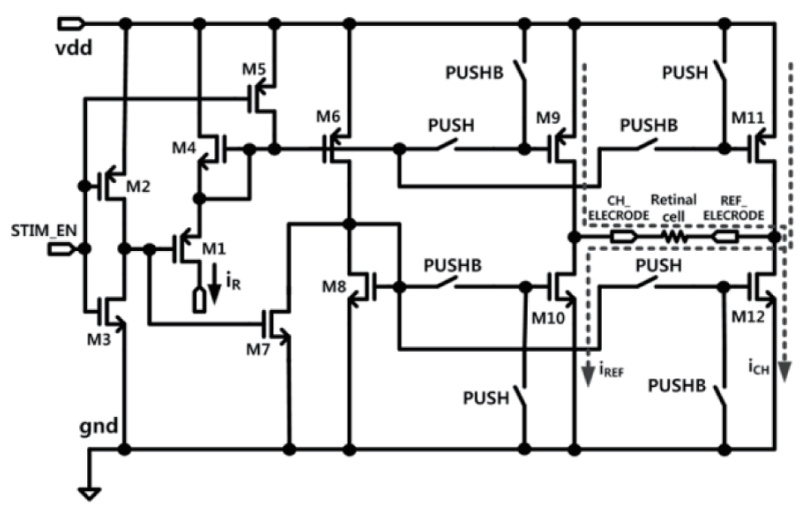

(b)

Fig. 6. Conventional circuit schematics. (a) Typical biphasic current stimulator. (b) Typical implementation of biphasic current driver. ${ }^{(54)}$

driver generates the biphasic currents $i_{C H}$ and $i_{R E F}$ when STIM_EN is ' $H$ '. The PUSH signal determines the direction of the stimulating current. When the PUSH is ' $\mathrm{H}$ ', the anodic current $i_{C H}$ flows from the channel electrode to the reference electrode. When PUSHB is ' $\mathrm{H}$ ', the cathodic current $i_{R E F}$ flows from the reference electrode to the channel electrode.

Current stimulators generally require high output voltage compliance, because the stimulators inject the biphasic current pulse with a maximum amplitude of several hundred $\mu \mathrm{A}$ into the microelectrodes and tissues of several tens of $\mathrm{k} \Omega$ impedance. For example, the output voltage compliance of $10 \mathrm{~V}(= \pm 5 \mathrm{~V})$ is required to drive $10 \mathrm{k} \Omega$ with a biphasic current pulse of $\pm 500 \mu \mathrm{A}$. Thus, the previous current stimulators are implemented using a high-voltage (HV) CMOS process, and adopt a static high supply voltage of 5 to $30 \mathrm{~V} .^{(55)}$

In our group, a current stimulator with an adaptive supply regulator was reported to enhance thermal safety. ${ }^{(55)}$ The internal power supply voltage is not static and adaptively regulates to the minimum required voltage for stimulation. Thus, current stimulation with lower voltage than the previous static HV stimulators can be achieved. The adaptive regulator provides the adaptively regulated internal supply voltage using a current feedback loop, as shown in Fig. 7. Additionally, the current feedback loop enhances the accuracy of the current amplitude and is robust to variations in the load impedances. The feedback loop in the regulator increases the internal supply voltage when the monitored current is smaller than the desired current, and reduces the internal supply voltage when the monitored current is higher than the desired current. Thus, the proposed stimulator can be operated with the minimum supply voltage, and potential damage to the tissues by HV stimulation can be avoided.

Also, to reduce the power consumption and to achieve the high-resolution scheme, a digitalto-analog converter (DAC) sharing scheme was reported, as shown in Fig. $8 .^{(54)}$ In the typical stimulator circuit, both the DAC and the biphasic electrode driver are included in the unit pixel circuits. However, our approach used a single shared DAC outside of the pixels. The output of the DAC is stored by the sampling switch and holding capacitor in the unit pixel. The pixel size can be reduced by sharing the DAC. As the number of channels is increased for high-resolution systems, the size reduction effect of the proposed DAC sharing scheme increases. The main specifications of the previously reported stimulators are summarized in Table 1. 


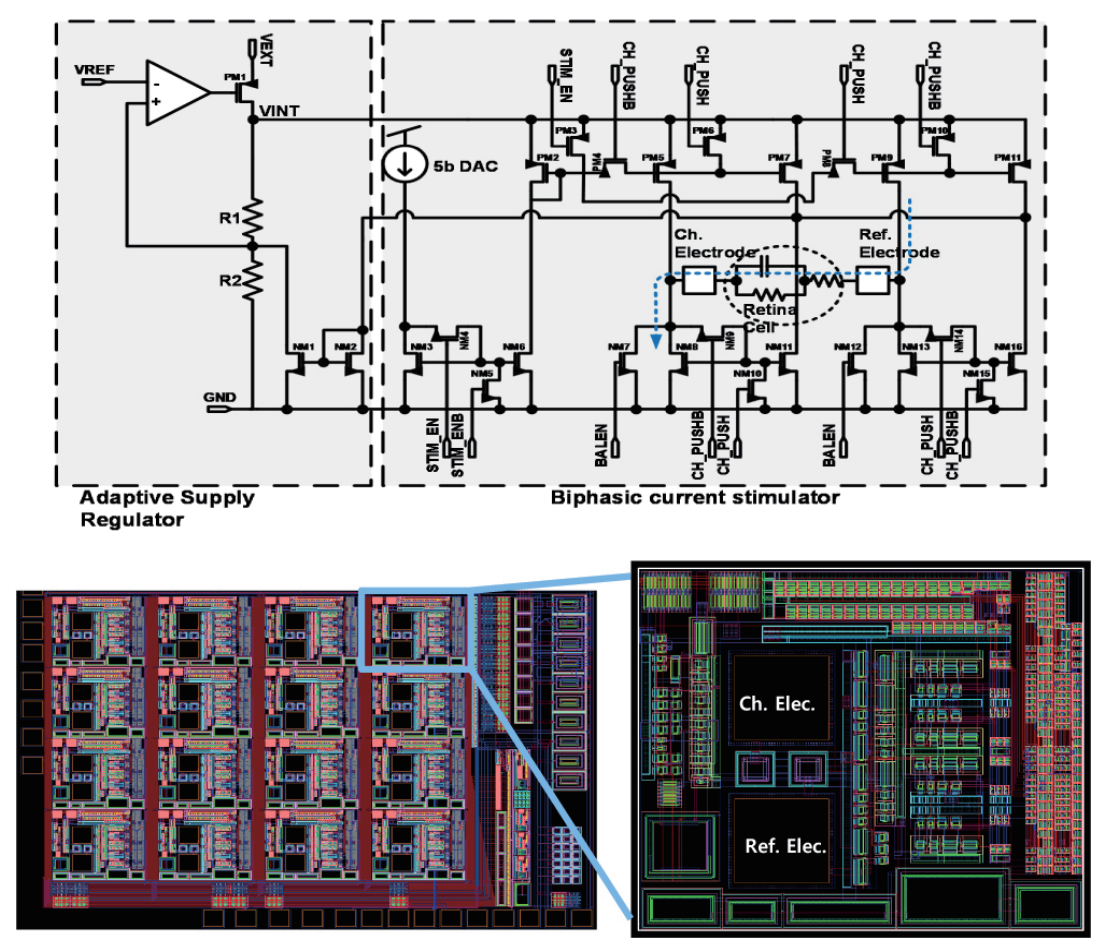

Fig. 7. (Color online) Adaptive supply regulator in stimulator.

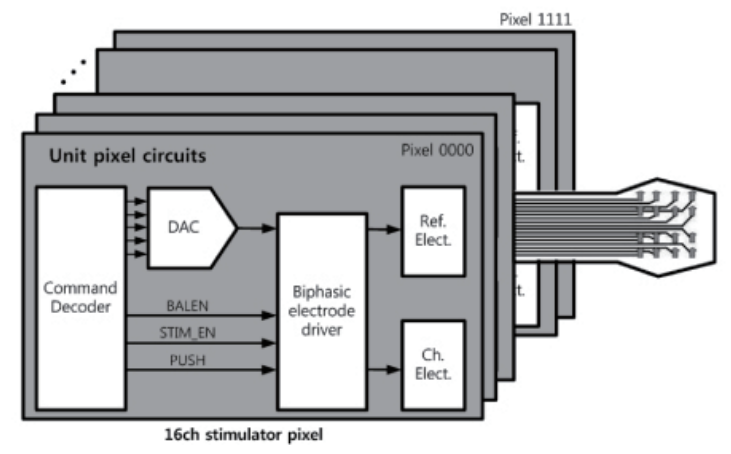

(a)

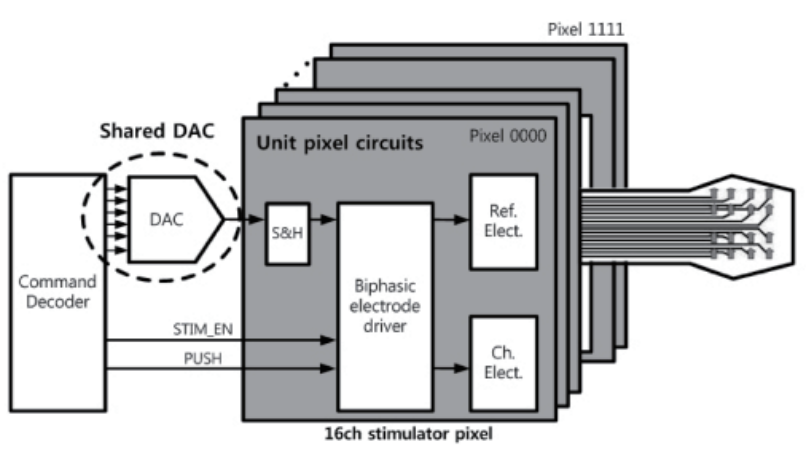

(b)

Fig. 8. Low power stimulator implementation using DAC sharing. ${ }^{(54)}$ (a) Typical scheme: one DAC per pixel. (b) DAC-sharing scheme: one DAC per stimulator.

Table 1

Performance of current stimulators.

\begin{tabular}{lcccc}
\hline & TBCAS 2014 & JSTS $2014^{(54)}$ & JBN 2013 & JSSC 2014 \\
\hline $\begin{array}{c}\text { Stimulation } \\
\text { frequency }\end{array}$ & $50 \mathrm{~Hz}$ & $625 \mathrm{~Hz}$ & $\begin{array}{c}1 \mathrm{kHz} \\
\text { (programmable) }\end{array}$ & N/A \\
\hline $\begin{array}{l}\text { Stimulation } \\
\text { current }\end{array}$ & $\begin{array}{c}\text { Up to } 1 \mathrm{~mA} \\
(5 \mathrm{bit})\end{array}$ & $\begin{array}{c}40 \mu \mathrm{A} \\
(6 \mathrm{bit})\end{array}$ & $\begin{array}{c}\text { Up to } 620 \mu \mathrm{A} \\
(5 \mathrm{bit})\end{array}$ & $\begin{array}{c}465 \mu \mathrm{A} \\
(5 \mathrm{bit})\end{array}$ \\
\hline $\begin{array}{l}\text { Pulse } \\
\text { duration }\end{array}$ & $\begin{array}{c}100 \mu \mathrm{s}-1 \mathrm{~ms} \\
(100 \mu \mathrm{s} \mathrm{step)}\end{array}$ & $1 \mathrm{~ms}$ & $\begin{array}{c}10 \mu \mathrm{s}-1 \mathrm{~ms} \\
(10 \mu \mathrm{s} \mathrm{step})\end{array}$ & N/A \\
\hline $\begin{array}{l}\text { Remarks } \\
\text { Charge balancing by } \\
\text { shorting }\end{array}$ & $\begin{array}{c}\text { DAC sharing, } \\
\text { arbitrary waveforms }\end{array}$ & $\begin{array}{c}\text { Adaptive supply regulator, } \\
\text { arbitrary waveforms }\end{array}$ & $\begin{array}{c}\text { Charge balancing by shorting, } \\
\text { fully integrated }\end{array}$ \\
\hline
\end{tabular}




\section{Animal Experiments}

\subsection{In vitro experiments}

To determine the optimum stimulation protocol for a subretinal prosthesis, retinal photoreceptor cells of rd1 mice are stimulated, and the responses of retinal ganglion cells were recorded in an in vitro environment. ${ }^{(57)}$ In this paper, we describe a novel sensing configuration for the retinal physiology analysis of the subretinal implant, in which the threshold charges of the bipolar/monopolar stimulation are in the range of 10-20 nC, as shown in Fig. 9(a).

Also, our group has developed a high-resolution retinal stimulation system based on CMOS image sensors (CISs) ${ }^{(15)}$ and SiNW photodetectors, ${ }^{(46)}$ and have conducted in vitro animal experiments. As shown in Ref. 15, the CIS-based stimulator IC generated biphasic current from 0 to $300 \mu \mathrm{A}$ as a function of incident light intensity. The electrically evoked ganglion cell responses in Fig. 9(b) show that more neural spikes are evoked as the stimulator IC injects a higher stimulation current, which can be controlled by adjusting the light intensity and charging time. The area of a single pixel in the proposed stimulator is only $50 \times 55 \mu \mathrm{m}^{2}$ including the photodiode, thus approximately 5000 pixels can be integrated in an MEA for high resolution. In Fig. 9(c), in vitro experimental results using a SiNW-based photodetection circuit is shown. The rectangular stimulation pulses are modulated to a stimulation current pulse via the SiNW photodetection circuit, which is proportional to light intensity, and is delivered to the target cell through the microelectrode.

\subsection{In vivo experiments}

Our group's arrowhead-shaped MEA consists of polyimide, titanium (Ti), and Au. ${ }^{(32)}$ These materials are well known as being biocompatible from previous research both in vitro and in vivo. ${ }^{(44,58,59)}$ In our in vivo biocompatibility examination of fabricated MEAs, New Zealand white rabbits were used. All procedures conformed to the Association for Research in Vision and Ophthalmology (ARVO) statement on the use of animals in ophthalmic and vision research. The fabricated MEA was implanted into a rabbit eye by the suprachoroidal approach. The in vivo stability of the implanted MEA was examined by the fundus image and optical coherent tomography (Cirrus OCT, Carl Zeiss, Dublin, CA, USA), as shown in Fig. 10(a). Fundus examination shows that choroidal vessels run over the inserted MEA, and the stimulation site is located near the visual streak. The OCT image 4 weeks after the insertion surgery reveals that the overlying retina and choroids are intact without any inflammation or swelling. In addition, in vivo electrical stimulation was performed, as shown in Fig. 10(b). The electrically evoked cortical potential (EECP) recording is performed simultaneously with biphasic current stimulation, and its waveform was compared with that of the visually evoked potential (VEP). 
(a)
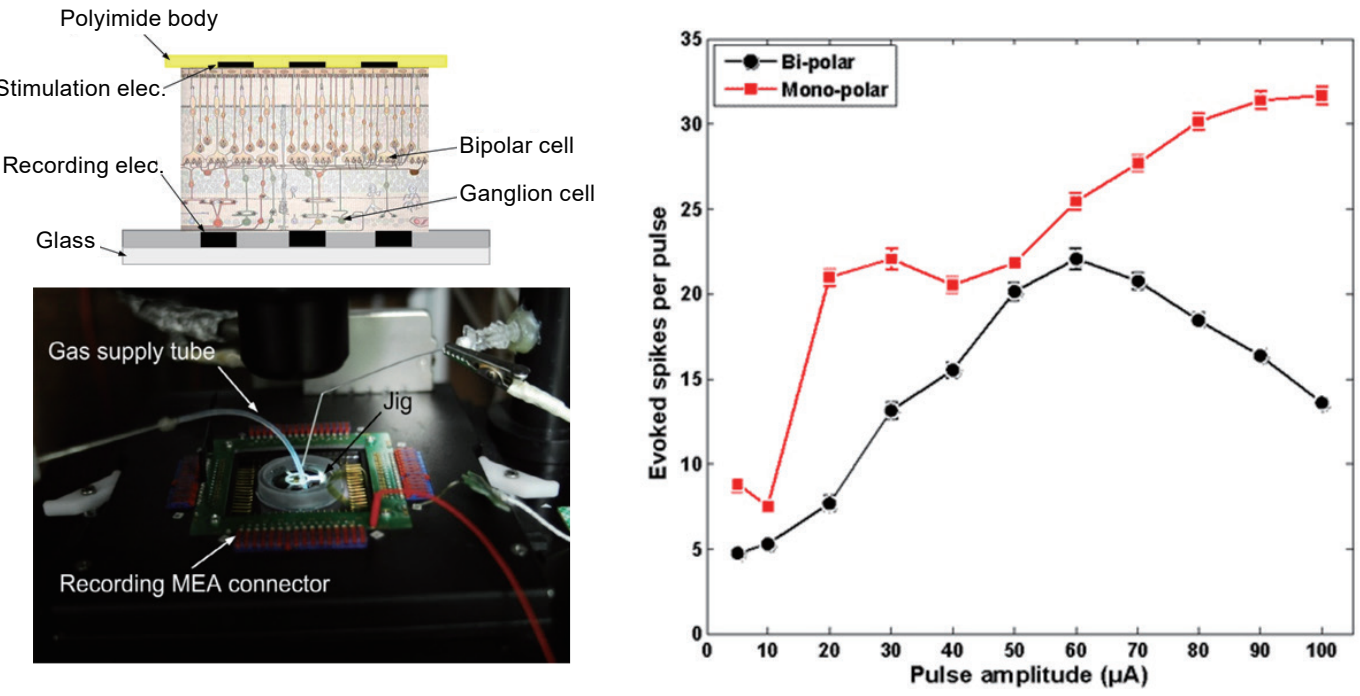

(b)
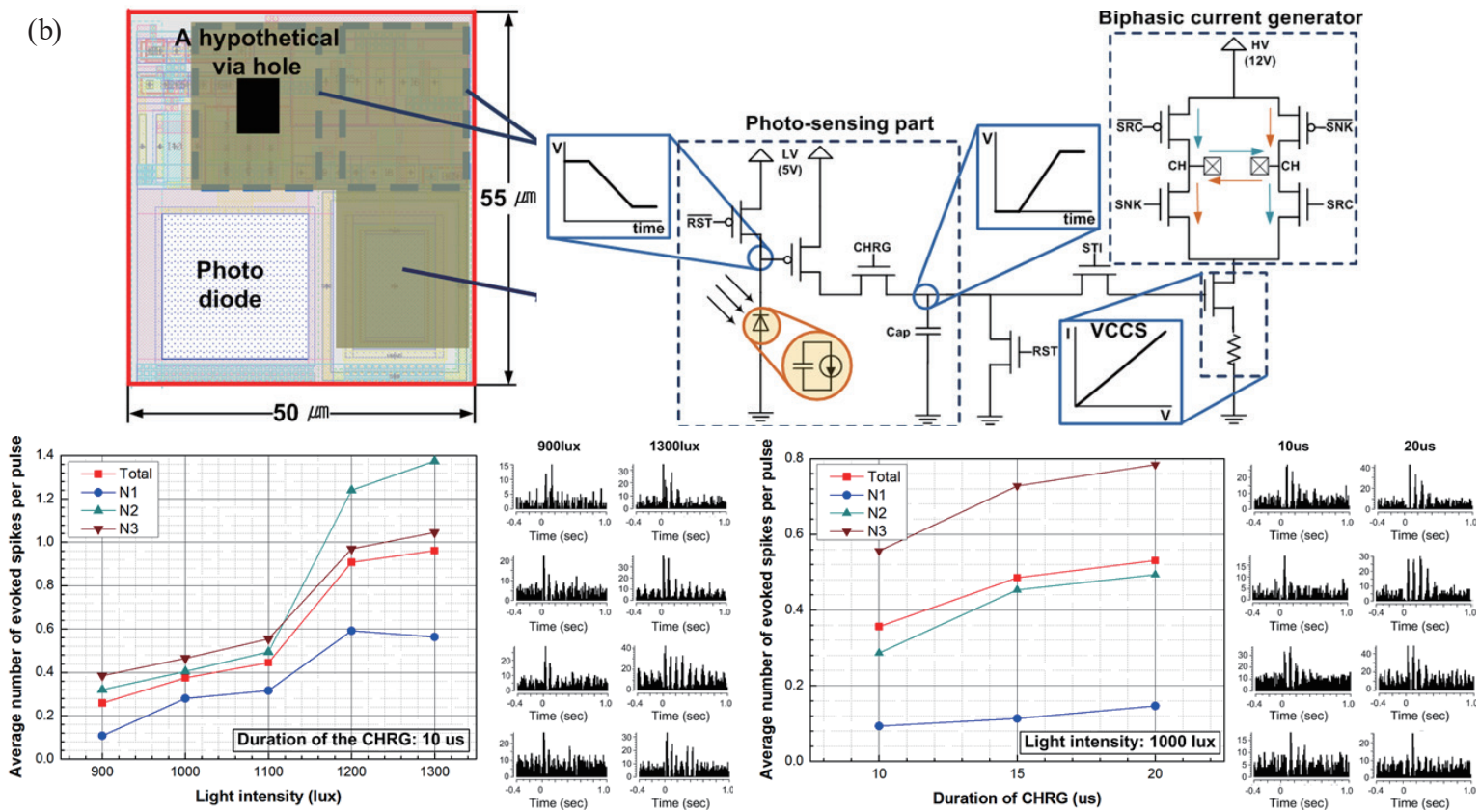

(c)
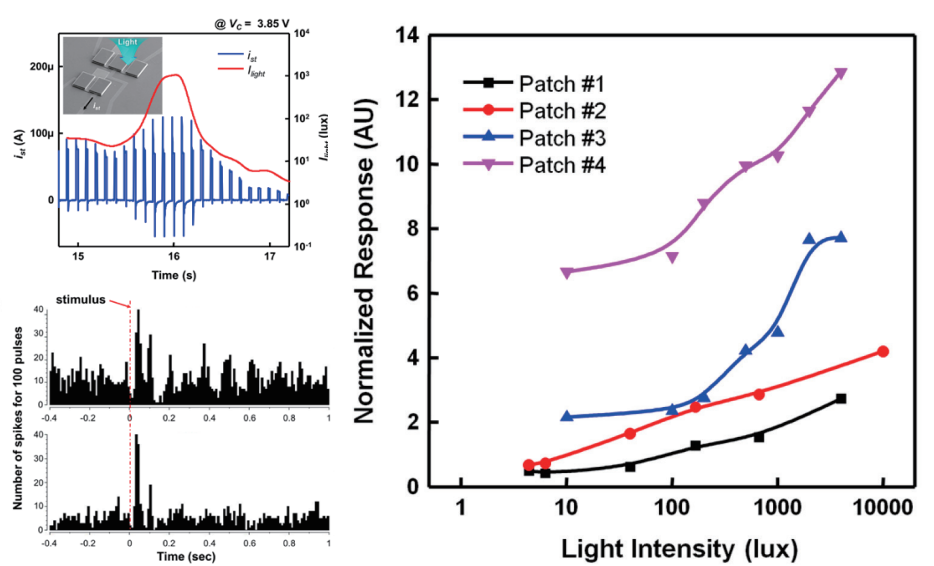

Fig. 9. In vitro animal experimental results. (a) Retinal physiology analysis for subretinal implant. ${ }^{\text {(57) }}$ (b) CISbased stimulation system. ${ }^{(15)}$ (c) SiNW-based stimulation system. ${ }^{(46)}$ 
(a)
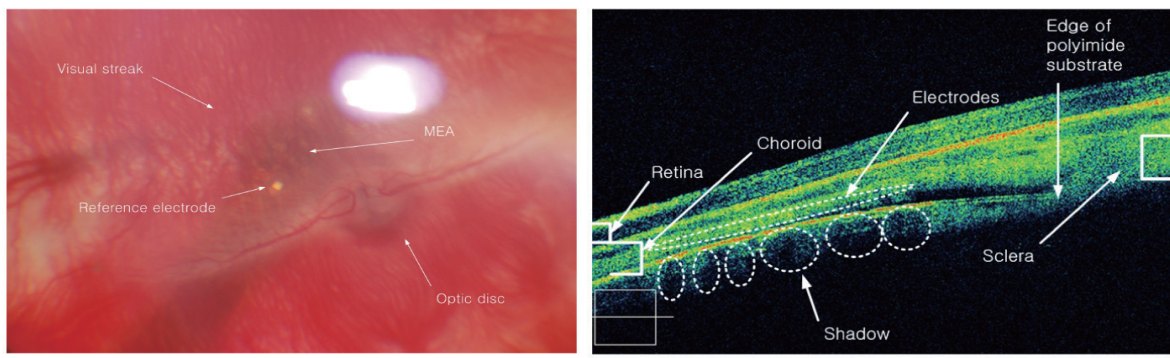

(b)
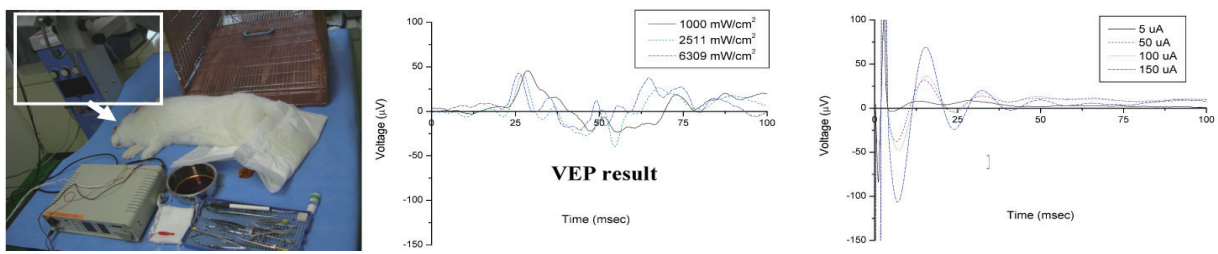

Fig. 10. (Color online) In vivo animal experimental results. ${ }^{(32)}$ EECP.

\section{Conclusions}

Retinal prosthetic devices are under development worldwide for the vision rehabilitation of the blind. To improve performance in daily life, a high-resolution device is essential, which requires highly collaborative research on MEAs, stimulation circuits, and signal processing. In this paper, the implementations of high-resolution retinal prosthetic systems reported by research groups worldwide are reviewed, including that of our group. Various design strategies, fabrication methods, system implementations, and animal experiments have been conducted to improve the performance of the system. Although some clinical trials have already started, there are still challenges to overcome for the successful implementation of retinal implants as a visual perception device for the blind.

\section{Acknowledgments}

This work was supported by the National Research Foundation of Korea (NRF) grant funded by the Korea government (MSIP; Ministry of Science, ICT \& Future Planning) (No. NRF-2017R1C1B5017561). This work was also supported by a grant to Bio-Mimetic Robot Research Center Funded by Defense Acquisition Program Administration, and by Agency for Defense Development (UD160027ID). The authors would like to thank Prof. Y. S. Goo, Dr. S. W. Jung, Dr. J. Ahn, Dr. S. K. Park, Dr. S. J. Hong, Dr. H. J. Yoo, S. Oh, and J. Y. Shin for their contribution to the retinal prosthetic system project

\section{References}

1 N. Congdon, B. O’Colmain, C. C. Klaver, R. Klein, B. Munoz, D. S. Friedman, J. Kempen, H. R. Taylor, and P. Mitchell: Arch. Ophthalmol. 122 (2004) 477. 
2 D. T. Hartong, E. L. Berson, and T. P. Dryja: The Lancet 368 (2006) 1795.

3 M. A. Zarbin: Arch. Ophthalmol. 122 (2004) 598.

4 K. Asakawa, H. Ishikawa, S. Uga, K. Mashimo, M. Kondo, and H. Terasaki: Neuro-Ophthalmol. 1 (2016) 1.

5 B. W. Jones, R. L. Pfeiffer, W. D. Ferrell, C. B. Watt, M. Marmor, and R. E. Marc: Exp. Eye Res. 150 (2016) 149.

6 M. M. Liu, J. Tuo, and C. C. Chan: Br. J. Ophthalmol. 95 (2011) 604.

7 D. Emerich and C. Thanos: Curr. Opin. Mol. Ther. 10 (2008) 506.

8 M. S. Humayun, E. De Juan, M. Del Cerro, G. Dagnelie, W. Radner, S. R. Sadda, and C. Del Cerro: Invest. Ophthalmol. Visual Sci. 41 (2000) 3100.

9 M. S. Humayun, E. de Juan Jr., and G. Dagnelie: Ophthalmology 123 (2016) S89.

10 J. F. Rizzo: J. Neuro-Ophthalomol. 31 (2011) 160.

11 J. U. Meyer: Sens. Actuators, A 97 (2002) 1.

12 E. Zrenner, K. U. Bartz-Schmidt, H. Benav, D. Besch, A. Bruckmann, V. P. Gabel, F. Gekeler, U. Greppmaier, A. Harscher, and S. Kibbel: Proc. Royal Society B: Biol. Sci. 278 (2011) 1489.

13 J. Seo, K. H. Kim, Y. Goo, K. Park, S. J. Kim, D. Cho, and H. Chung: Sens. Mater. 24 (2012) 153.

14 A. Pérez Fornos, J. Sommerhalder, A. Pittard, A. B. Safran, and M. Pelizzone: Vision Res. 48 (2008) 1705.

15 S. Oh, J. Ahn, S. Lee, H. Ko, J. -M Seo, Y. S. Goo, and D. Cho: IEEE Trans. Biomed. Eng. 62 (2015) 70.

16 S. Lee, J. Ahn, J.-M. Seo, H. Chung, and D. Cho: Sensors 15 (2015)14345.

17 G. E. Legge, S. J. Ahn, T. S. Klitz, and A. Luebker: Vision Res. 37 (1997) 1999.

18 J. Sommerhalder, E. Oueghlani, M. Bagnoud, U. Leonards, A. B. Safran, and M. Pelizzone: Vision Res. 43 (2003) 269.

19 K. Cha, K. W. Horch, and R. A. Normann: Vision Res. 32 (1992) 1367.

20 D. R. Geruschat, K. A. Turano, and J. W. Stahl: Optometry Vision Sci. 75 (1998) 525.

21 X. Cao, X. Sui, Q. Lyu, L. Li, and X. Chai: J. NeuroEng. Rehabil. 12 (2015) 73.

22 M. O. Heuschkel, M. Fejtl, M. Raggenbass, D. Bertrand, and P. Renaud: J. Neurosci. Methods 114 (2002) 135.

23 R. Wang, X. Huang, G. Liu, W. Wang, F. Dong, and Z. Li: J. Microelectromech. Syst. 19 (2010) 367.

24 S. Rajaraman, M. A. McClain, S. Choi, J. D. Ross, S. P. DeWeerth, M. C. LaPlaca, and M. G. Allen: Proc. 2007 Int. Conf. Solid-State Sensors, Actuators and Microsystems, Lyon, France, 10-14 June, (2007) 12511254.

25 R. Bhandari, S. Negi, L. Rieth, R. A. Normann, and F. Solzbacher: Proc. 2007 Int. Conf. Solid-State Sensors, Actuators and Microsystems, Lyon, France, 10-14 June, (2007) 1231-1234.

26 K. Hungar, M. Görtz, E. Slavcheva, G. Spanier, C. Weidig, and W. Mokwa: Sens. Actuators, A 123-124 (2005) 172.

27 E. T. Kim, J. M. Seo, S. J. Woo, J. A. Zhou, H. Chung, and S. J. Kim: Sensors 8 (2008) 5845.

28 D. Scribner, M. Humayun, B. Justus, C. Merritt, R. Clein, J. G. Howard, M. Peckerar, F. Perkins, L. Johnson, W. Bassett, P. Skeath, E. Margalit, K. A. Eong, J. Weiland, E. de Juan Jr., J. Finch, R. Graham, C. Trautfield, and S. Taylor: Proc. 23rd Annu. Int. Conf. IEEE Engineering in Medicine and Biology Society, Istanbul, Turkey, October 25-28, (2001) 3430-3435.

29 M. Kusko, F. Craciunoiu, B. Amuzescu, F. Halitzchi, T. Selescu, A. Radoi, M. Popescu, M. Simion, A. Bragaru, and T. Ignat: Sensors 12 (2012) 16571.

30 M. Djilas, C. Oles, H. Lorach, A. Bendali, J. Degardin, E. Dubus, G. Lissorgues-Bazin, L. Rousseau, R. Benosman, S.-H. Leng, S. Joucla, B. Yvert, P. Bergonzo, J. Sahel, and S. Picaud: J. Neural Eng. 8 (2011) 045020.

31 Z. Xiang, J. Liu, and C. Lee: Microsyst. Nanoeng. 2 (2016) 16012.

32 K. Koo, S. Lee, S. H. Bae, J.-M. Seo, H. Chung, and D. Cho: J. Microelectromech. Syst. 20 (2011) 251.

33 K. Koo, H. Chung, Y. Yu, J. Seo, J. Park, J.-M. Lim, S.-J. Paik, S. Park, H. M. Choi, M.-J. Jeong, G. S. Kim, and D. Cho: Sens. Actuators, A 130-131 (2006) 609.

34 S.-J Paik, Y. Park, and D. Cho: IOP J. Micromechanics and Microeng. 13 (2003) 373.

35 S. Byun, J.-M. Lim, S.-J. Paik, A. Lee, K. Koo, S. Park, J. Park, B.-D. Choi, J. M. Seo, K. Kim, H. Chung, S. Y. Song, D. Jeon, and D. Cho: IOP J. Micromechanics and Microeng. 15 (2005) 1279.

36 S. Lee, J. Ahn, H. Yoo, S. Jung, S. Oh, S. Park, and D. Cho: Proc. 35th Annu. Int. Conf. IEEE Engineering in Medicine and Biology Society, Osaka, Japan, 3-7 July (2013) 3535-3538.

37 S. J. Hong, S. Lee, J. Ahn, S. K Park, K. Koo, and D. Cho: Proc. 16th Int. Conf. Solid-State Sensors, Actuators and Microsystems, Beijing, China, June 5-9 (2011) 202-205.

38 S. Lee, S. W. Jung, H. J. Yoo, S. J. Oh, and D. Cho: IOP J. Micromech. Microeng. 24 (2014) 075018.

39 M. S. Humayun, J. D. Dorn, L. da Cruz, G. Dagnelie, J. Sahel, P. E. Stanga, A. V. Cideciyan, J. L. Duncan, D. Eliott, E. Filley, A. C. Ho, A. Santos, A. B. Safran, A. Arditi, L. V. Del Priore, R. J. Greenberg, and Argus II Study Group: Ophthalmology 119 (2012) 779. 
40 S. Klauke, M. Goertz, S. Rein, D. Hoehl, U. Thomas, R. Eckhorn, F. Bremmer, and T. Wachtler: Invest. Ophthalmol. Visual Sci. 52 (2011) 449.

41 A. Y. Chow, A. K. Bittner, and M. T. Pardue: Trans. Am. Ophthalmol. Soc. 108 (2010) 120.

42 S. Lee, S. W. Jung, J. Ahn, H. J. Yoo, S. K. Park, and D. Cho: Proc. 17th Int. Conf. Solid-State Sensors, Actuators and Microsystems, Barcelona, Spain, June 16-20, (2013) 673-676.

43 J. Ahn, S. Lee, S. J. Hong, H. J. Yoo, S. W. Jung, S. K. Park, K. Ko, and D. Cho: J. Biomed. Nanotechnol. 9 (2013) 621.

44 S. J. Bae, J. H. Che, J.-M. Seo, J. Jeong, E. T. Kim, S. W. Lee, K. Koo, G. J. Suaing, N. H. Lovell, D. Cho, S. J. Kim, and H. Chung: Invest. Ophthalmol. Visual Sci. 53 (2012) 2653.

45 S. Lee, S. W. Jung, S. K. Park, J. Ahn, S. J. Hong, H. J. Yoo, M. H. Lee, and D. Cho: Sens. Mater. 24 (2012) 205.

46 S. W. Jung, J. Shin, K. Pi, Y. S. Goo, and D. Cho: Sensors 16 (2016) 2035.

47 S. Lee, S. Park, and D. Cho: J. Microelectromech. Syst. 8 (1999) 409.

48 J. Y. Shin, K. Pi, S. Jung, and D. Cho: Proc. IEEE 29th Int. Conf. Micro Electro Mechanical Systems (MEMS 2016), Shanghai, China, January 24-28 (2016) 469-472.

49 S. Lee, S. W. Jung, S. Park, J. Ahn, S. J. Hong, H. J. Yoo, M. H. Lee, and D. Cho: Proc. IEEE 25th Int. Conf. Micro Electro Mechanical Systems (MEMS 2012), Paris, France, January 29-Faburary 2 (2012) 1364-1367.

50 A. Butterwick, A. Vankov, P. Huie, Y. Freyvert, and D. Palanker: IEEE Trans. Biomed. Eng. 54 (2007) 2261.

51 V. Singh, A. Roy, R. Castro, K. McClure, R. Dai, R. Agrawal, R. Greenberg, J. Weiland, M. Humayun, and G. Lazzi: IEEE Trans. Biomed. Circuits and Syst. 2 (2008) 289.

52 H. Chun, Y. Yang, and T. Lehmann: IEEE Trans. Biomed. Eng. 8 (2014) 108.

53 C. Q. Huang, R. K. Shepherd, P. M. Center, P. M. Seligman, and B. Tabor: IEEE Trans. Biomed. Eng. 46 (1999) 461.

54 C. Seok, H. Kim, S. Im, H. Song, K. Lim, Y. S. Goo, K. Koo, D. Cho, and H. Ko: J. Semicond. Technol. Sci. 14 (1999) 658.

55 H. Ko, S. Lee, J. Ahn, S. J. Hong, H. J. Yoo, S. W. Jung, S. K. Park, and D. Cho: J. Biomed. Nanotechnol. 9 (2013) 992.

56 N. Tran, S. Bai, J. Yang, H. Chun, O. Kavehei, Y. Yang, V. Muktamath, D. Ng, H. Meffin, M. Halpern, and E. Skafidas: IEEE J. Solid-State Circuits 49 (2014) 751.

57 K. Koo, S. Lee, J. H. Yee, S. B. Ryu, K. H. Kim, Y. S. Goo, and D. Cho: Sensors 12 (2012) 3131.

58 J.-M. Seo, S. J. Kim, H. Chung, E. T. Kim, H. G. Yu, and Y. S. Yu: Mater. Sci. Eng., C 24 (2004) 185.

59 J. A. Zhou, S. J Woo, S. I. Park, E. T. Kim, J.-M. Seo, H. Chung, and S. J. Kim: J. Biomed. Biotechnol. 2008 (2008) 547428. 